www.volsu.ru

DOI: https://doi.org/10.15688/jvolsu11.2017.3.5

UDC 502.51:581.526.325

LBC 26.221

\title{
OPTIMIZATION OF PROCESSES OF EMISSION OF VOC FROM OCEAN TO ATMOSPHERE IN PERIOD OF FLOURISHING OF PHYTOPLANCTONES
}

\author{
Hikmet Gamid ogly Asadov \\ National aerospace Agency, Baku, Azerbaijan \\ Sima Azhdar gyzy Askerova \\ National aerospace Agency, Baku, Azerbaijan
}

\begin{abstract}
It is well-known that emission of methanol and dimethilsulfide (DMS) from surface of ocean depends on quantity of photosynthetic active radiation (PAR) and some biological parameters. Therefore PAR and biological factors should be used upon estimation of effect of oceans on balance of volatile organic compaunds in atmosphere. The major factors impacting on emission of osoprene to atmosphere os wind speed and intensity of photosyntheticaly active light. In the article the questions on optimum choice of some external effecting factors are considered upon which the minimization of total emission of isoprene to atmosphere taking into account accepted model interrelations would be possible. Some models of emission of isoprene to atmosphere possessing the extremum feature that is the minimum of integral value of emitted isoprene in some interval of quantity of photo synthetically active optical radiation of the Sun upon keeping the revealed optimum functions of interrelations between intensity of optical radiation and wind speed are suggested. The significant non- coincidence of these functions for both the considered models of isoprene emission from ocean water to atmosphere upon some limitation condition applied for searched for optimum function is revealed. Such difference in revealed optimum emission regimes stressed out necessity for further modification of mathematical models of VOC emitted from oceanic surface.
\end{abstract}

Key words: optimization, emission, ocean waters, isoprene, correlation, atmosphere.

УДК 502.51:581.526.325

ББК 26.221

\section{ОПТИМИЗАЦИЯ ПРОЦЕССОВ ЭМИССИИ ЛЕТУЧИХ ОРГАНИЧЕСКИХ СОЕДИНЕНИЙ ИЗ ОКЕАНА В АТМОСФЕРУ В ПЕРИОД ЦВЕТЕНИЯ ФИТОПЛАНКТОНОВ}

\author{
Хикмет Гамид оглы Асадов \\ Национальное аэрокосмическое агентство, г. Баку, Азербайджан \\ Сима Аждар гызы Аскерова \\ Национальное аэрокосмическое агентство, г. Баку, Азербайджан
}

\begin{abstract}
Аннотация. Известно, что эмиссии метанола, ацетона, ацетальдегида, изопрена и диметилсульфида (DMS) из поверхности океана зависит от величины фотосинтетически активной радиации (PAR) и некоторых биологических параметров. Следовательно, PAR и биологические факторы должны быть использованы при оценке влияния океанов на баланс летучих органических соединений в атмосфере. Важнейшими факторами, влияющими на эмиссию изопрена в атмосферу являются скорость ветра и интенсивность фотосинтетически активного света. С учетом вышесказанного в статье рассматриваются вопросы оптимального выбора некоторых внешне воздействующих факторов при котором можно было бы минимизировать суммарную эмиссию изопрена в атмосферу с учетом принятых модельных соотношений.
\end{abstract}




\section{ЭКОЛОГИЯ И ПРИРОДОПОЛЬЗОВАНИЕ}

Предложены модели эмиссии изопрена в атмосферу обладающие экстремальным свойством, т.е. минимумом интегрального значения эмитированного изопрена в некотором интервале величин фотосинтетически активного оптического излучения Солнца при соблюдении выявленных оптимальных функций взаимосвязи между интенсивностью оптического излучения и скоростью ветра. Выявлено существенное несовпадение видов этих функций для двух рассмотренных моделей процесса эмиссии изопрена из океанических вод в атмосферу при наложении некоторого фиксированного функционального ограничения на искомую оптимальную функцию. Такое различие в выявленных оптимальных режимах эмиссии указывает на необходимость дальнейшего усовершенствования математических моделей потоков летучих органических соединений, исходящих из океанических вод.

Ключевые слова: оптимизация, эмиссия, океанические воды, изопрен, корреляция, атмосфера.

Как сообщается в работе [1], процессы эмиссии из океана летучих органических соединений в атмосферу изучены не полностью. Согласно [1], океанические эмиссии метанола, ацетона, ацетальдегида, изопрена и диметилсульфида (DMS) зависит от величины фотосинтетически активной радиации (PAR) и некоторых биологических параметров. Исследования на границе океан - атмосфера, проведенные в [1] показали, что после цветения фитопланктонов летучие органические соединения, измеренные протонным масс - спектрометром (PTR-MS) в небольшой замкнутой акватической экосистеме (mesocosm) имели следующую закономерность: изопрен и асетальдегид эмитировался в количестве, хорошо коррелирующимся количеством света $\left(\mathrm{r}_{\text {AVCORR,ISOPRENE }}=0,49 ; \mathrm{r}_{\text {AVCORR, ASETALDEHYDE }}=\right.$ $0,70)$, а также количеством фитопланктонов. DMS также эмитируется в атмосферу, однако количество DMS значительно не коррелирует величиной света $\left(\mathrm{r}_{\mathrm{AVCORR}, \mathrm{DMS}}=0,01\right)$.

Исследования, проведенные в [1], показывают, что PAR и биологические факторы должны быть использованы при оценке влияния океанов на баланс летучих органических соединений в атмосфере.

Прежде всего отметим, что изопрен $(\mathrm{CH} 2=\mathrm{CH}-\mathrm{C}(\mathrm{CH} 3)=\mathrm{CH} 2)$ является биогеничным углеводородом, эмитируемым деревьями, травой и океаническими фитопланктонами. Целью эмиссии является противодействие абиотическому стрессу. Изопрен играет важную роль в формировании атмосферного озона, аэрозолей и облаков.

Согласно работе [4, 5, 8, 9, 11], существует корреляционная связь между изопреном и концентрацией хлорофилла $a$ в морской воде. Графически такая корреляционная связь отображена на рисунке 1 [3]. Как видно из графиков, приве- денных на рисунке 1 максимум концентрации изопрена находится на более низкой глубине, чем максимум концентрации хлорофилла.

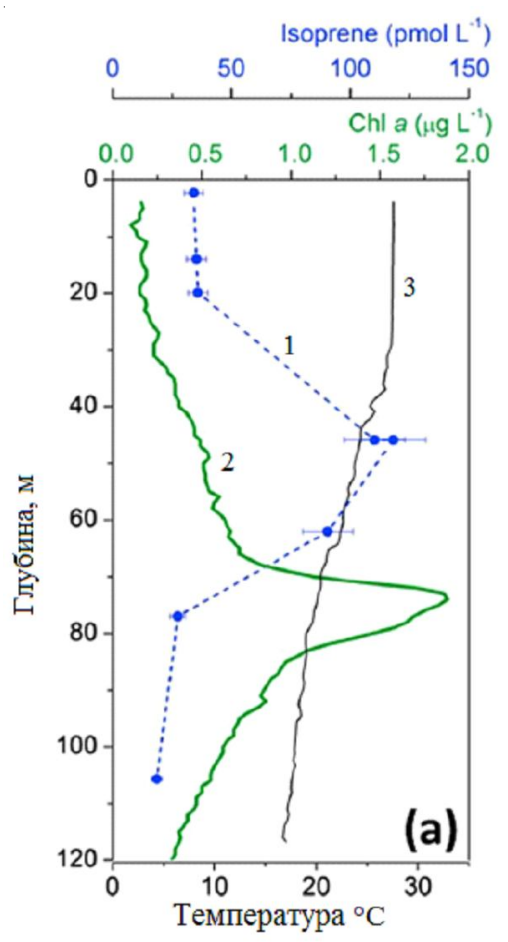

Рис. 1. Графическое отображение наличия корреляционной связи между изопреном и хлорофиллом в океанской воде в зависимости от глубины и температуры. Цифрами указаны:

1 - кривая изменения концентрации изопрена по глубине;

2 - кривая изменения концентрации хлорофилла по глубине; 3 - кривая изменения температуры воды по глубине [3]

Согласно [3], справедливы следующие регрессионные уравнения между количеством изопрена и концентрацией Chl в морской воде

$$
\begin{aligned}
& I S O=37,9 \cdot[\text { Chla }]+17,5 ; R^{2}=0,37 \\
& I S O=15,1 \cdot[\text { Chla }]+18,4 ; \quad R^{2}=0,55
\end{aligned}
$$


Согласно работе [10], существует прямая корреляция между концентрациями изопрена и хлорофилла в воде (рис. 2), зависящая от сезона. Так, для Северного моря в июне $\mathrm{r}^{2}=0,62$, а в апреле $\mathrm{r}^{2}=0,43$.

Согласно работе [7], важнейшими факторами, влияющими на эмиссию изопрена в атмосферу являются скорость ветра и интенсивность фотосинтетически активного света (400 - 800 нм).

На рисунках 3 и 4 показаны соответствующие скатерограммы зависимости концентрации изопрена в атмосфере над Средиземным морем. Согласно [3], при скорости ветра ниже 4 м/с концентрация изопрена уменьша- ется по экспоненциальному закону, а при значениях выше 4 м/с не изменяется (рис. 3). Зависимость концентрации изопрена в атмосфере над морем от интенсивности света также экспоненциальна (рис. 4).

В настоящее время существуют несколько математических моделей, отображающих процесс эмиссии в атмосферу морского изопрена. Например, согласно модели, изложенной в [6], эмиссионный поток может быть вычислен по формуле

$$
F_{V O C}=k_{w}(U, S S T) \cdot C h l a \cdot b,
$$

где $\mathrm{k}_{\mathrm{w}}$ - коэффициент передачи изопрена, зависящая от скорости ветра U и поверхностной темпе-

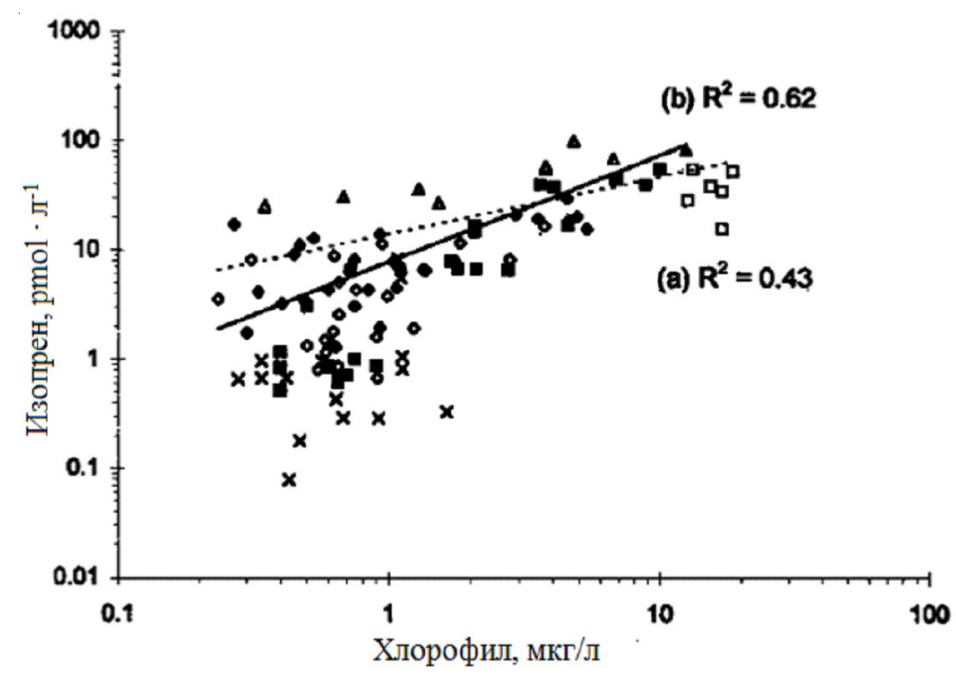

Рис. 2. Концентрация изопрена в морской воде как функция концентрации хлорофилла в Северном Море (июнь - обозначены треугольниками), (апрель - обозначены полыми ромбами), (декабрь - обозначены крестиками), а также в море Беллингкаузена (черные ромбы), в береговой зоне Северного моря (полые квадраты)

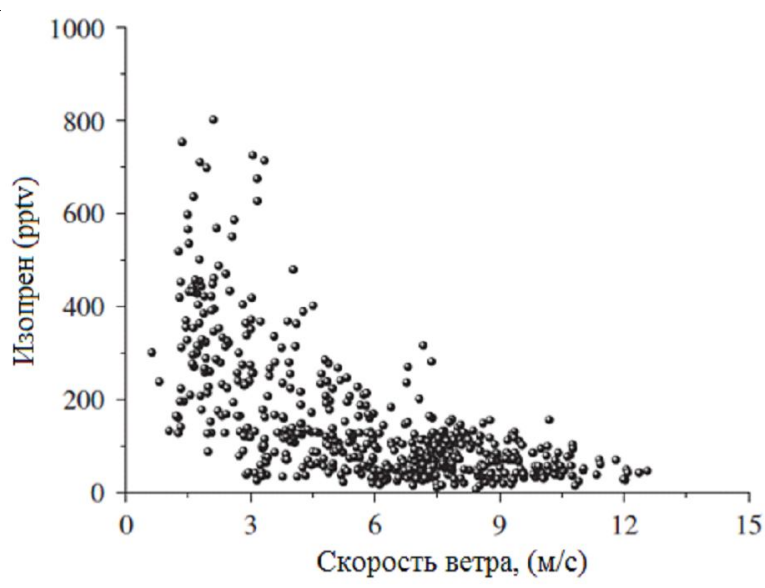

Рис. 3. Зависимость концентрации изопрена над морем от скорости ветра

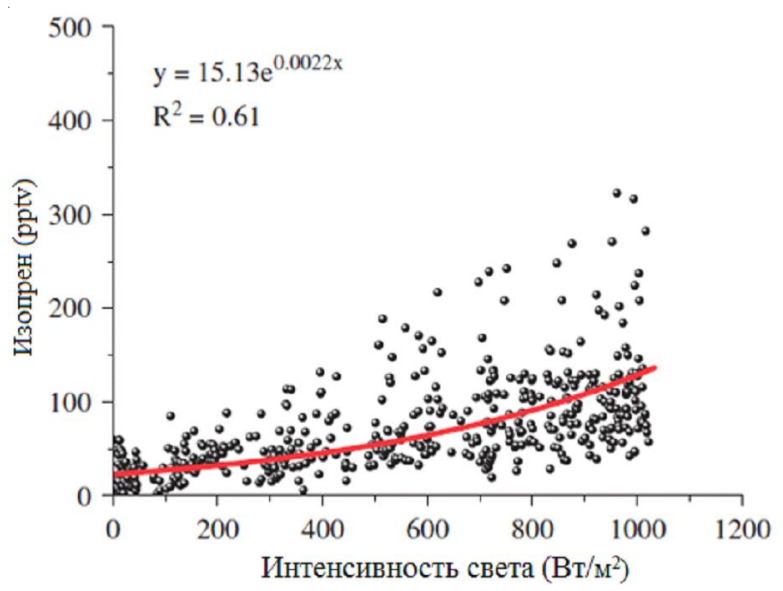

Рис. 4. Зависимость концентрации изопрена над морем от интенсивности света 


\section{ЭКОЛОГИЯ И ПРИРОДОПОЛЬЗОВАНИЕ}

ратуры моря; b - коэффициент пропорциональности; Chla - концентрация хлорофилла $a$ в морской воде.

Согласно работе [2], общее колонное количество эмитированного в атмосферу изопрена $\mathrm{E}_{\text {ISO }}$ может быть вычислено по следующей формуле

$$
E_{I S O}=S A \cdot H_{\max } \cdot C h l a \cdot F_{I S O} \cdot \int_{0}^{H_{\max }} P d h
$$

где SA - площадь поверхности рассматриваемого участка, ${ }^{2} ; \mathrm{F}_{\text {ISO }}$ - эмиссионная фракция, то есть фракция водной колонны, эмитирующей изопрен; $\mathrm{H}_{\max }$ - общая глубина водяного слоя, способного продуцировать изопрен.

$$
H_{\text {max }}=\left(-\ln \left(\frac{2,5}{I_{0}}\right) \cdot k_{490}^{-1}\right)
$$

где: $\mathrm{I}_{0}$ - интенсивность солнечной радиации; $k_{490}^{-1}-$ коэффициент диффузного ослабления в воде.

Далее, в настоящей статье рассматриваются вопросы оптимального выбора таких внешне воздействующих факторов как $\mathrm{I}_{0}$ и U, при котором можно было бы минимизировать суммарную эмиссию изопрена в атмосферу с учетом моделей (3) и (4). Модель (3) с учетом графиков, представленных на рис. 3 и 4 при SST = const можно представить в виде

$$
F_{\text {VOC. } m}=k \cdot e^{-\frac{U}{a_{1}}} \cdot e^{a_{2} \cdot I_{0}} \cdot \text { Chla, }
$$

где $\mathrm{k}=$ const; $\mathrm{U}$ - скорость ветра; $\mathrm{a}_{1}=$ const; $\mathrm{a}_{2}=$ const. имеем

Интегрируя (6) в пределах $\mathrm{I}_{0}=0-\mathrm{I}_{0 \max }$

$$
F_{\text {VOС.m.uн }}=\int_{0}^{I_{0 \max }} k \cdot e^{-\frac{U}{a_{1}}} \cdot e^{a_{2} \cdot I_{0}} \cdot C h l a \cdot d I_{0}
$$

Так как исследуется оптимальная взаимосвязь параметров $\mathrm{U}_{\text {и }} \mathrm{I}_{0}$, при которой $F_{V O C . m . и н}$ достиг бы минимума, в функционал (7) вводим функцию $\mathrm{U}=\mathrm{U}\left(\mathrm{I}_{0}\right)$, на которую налагается следующее ограничительное условие

$$
F_{01}=\int_{0}^{I_{0 \max }} U\left(I_{0}\right) d I_{0}=C_{1} ; C_{1}=\text { const },
$$

В простейшем случае условие (8) подразумевает наличие функций $\mathrm{U}\left(\mathrm{I}_{0}\right)$ в виде

$$
\begin{aligned}
& U_{1}=U_{01}+k_{1} I_{0} \\
& U_{2}=U_{02}-k_{2} I_{0}
\end{aligned}
$$

где $\mathrm{U}_{01}, \mathrm{U}_{02}, \mathrm{k}_{1}, \mathrm{k}_{2}=$ const.

Общий вид функций (9), (10) показан на рисунке 5.

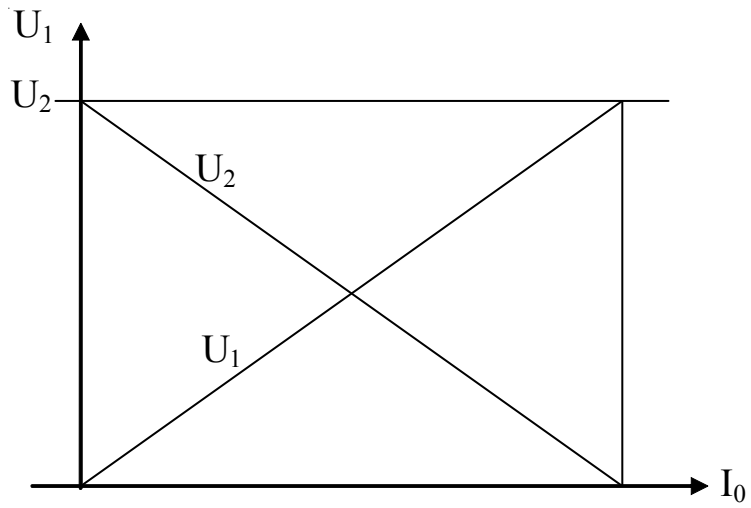

Рис. 5. Общий вид функций $\mathrm{U}_{1}$ и $\mathrm{U}_{2}$ определенных выражениями (9) и (10)

С учетом выражений (7) и (8) можно сформировать следующий функционал безусловной вариационной оптимизации

$$
F_{0}=\int_{0}^{I_{0 \max }} k \cdot e^{-\frac{U\left(I_{0}\right)}{a_{1}}} \cdot e^{a_{2} \cdot I_{0}} \cdot C h l a \cdot d I_{0}+\lambda\left[\int_{0}^{I_{\max }} U\left(I_{0}\right) d I_{0}-C_{1}\right](11)
$$

где $\lambda$ - множитель Лагранжа.

Согласно уравнению Эйлера решение вышеуказанной оптимизационной задачи на экстремум должно удовлетворить условию

$$
\frac{d\left\{k \cdot e^{-\frac{U\left(I_{0}\right)}{a_{1}}} \cdot e^{a_{2} \cdot I_{0}} \cdot C h l a+\lambda\left[U\left(I_{0}\right)-C_{1}\right]\right\}}{d U\left(I_{0}\right)}=0
$$

С учетом выражений (8), (12) можно показать, что минимальное значение $\mathrm{F}_{0}$ обеспечивается при линейной зависимости между $\mathrm{U}$ и $\mathrm{I}_{0}$, то есть

$$
\mathrm{U}=\mathrm{C}_{2} \mathrm{I}_{0}+\mathrm{C}_{3},
$$

где $\mathrm{C}_{2}, \mathrm{C}_{3}=$ const 
Решим аналогичную оптимизационную задачу применительно к модели (4) и (5). С учетом выражений (4) и (5) имеем

$$
E_{\text {ISO }}=S A \cdot \ln \left(\frac{I_{0}}{2,5}\right) \cdot k_{490}^{-1} \cdot C h l a \cdot F_{I S O} \cdot P \cdot H_{\max }(13)
$$

При выводе формулы (13) принято упрощающее предположение о том, что Р не зависит от $\mathrm{h}$.

С учетом информации, представленной на рис. 3 функционал (13) модифицируем в следующем виде

$$
E_{I S O, m}=e^{-\frac{U\left(I_{0}\right)}{a_{1}}} \cdot S A \cdot \ln \left(\frac{I_{0}}{2,5}\right) \cdot k_{490}^{-1} \cdot C h l a \cdot F_{I S O} \cdot P \cdot H_{\max }(14)
$$

Выражение (14), при перепишем как

$$
E_{I S O . m}=C_{4} \cdot e^{-\frac{U\left(I_{0}\right)}{a_{1}}} \cdot \ln \left(\frac{I_{0}}{2,5}\right) \cdot \text { Chla }
$$

где $\mathrm{C}_{4}=$ const.

На основе выражения (15) сформируем целевой функционал

$$
E_{I S O \text {.m. in }}=\int_{I_{0 . \text { min }}}^{I_{0 . \max }} C_{4} \cdot e^{-\frac{U\left(I_{0}\right)}{a_{1}}} \cdot \ln \left(\frac{I_{0}}{2,5}\right) \cdot C h l a \cdot d I_{0}
$$

С учетом выражений (8) и (16) сформируем оптимизационную задачу безусловной вариационной оптимизации.

$$
\begin{aligned}
E_{I S O \text {.m. } 0}= & \int_{I_{0 \text {. min }}}^{I_{\text {maxx }}} C_{4} \cdot e^{-\frac{U\left(I_{0}\right)}{a_{1}}} \cdot \ln \left(\frac{I_{0}}{2,5}\right) \cdot \operatorname{Chla} \cdot d I_{0}+ \\
& +\lambda\left[\int_{I_{0 . \text { min }}}^{I_{0 \text { max }}} U\left(I_{0}\right) d I_{0}-C_{1}\right]
\end{aligned}
$$

Можно показать, что при оптимальной зависимости

$$
U\left(I_{0}\right)=\ln \left[C_{5} \cdot \ln \left(\frac{I_{0}}{2,5}\right) \cdot \text { Chla }\right]
$$

где $\mathrm{C}_{5}=$ const

функционал (17) достигает минимума.

Таким образом, обе рассмотренные модели эмиссии изопрена в атмосферу обладают экстремальным свойством, точнее говоря минимумов интегрального значения эмитированного изопрена в некотором интервале величин фотосинтетически активного оптического излучения Солнца при соблюдении выявленных оптимальных функций взаимосвязи между интенсивностью оптического излучения и скоростью ветра. При этом выявлено существенное несовпадение видов этих функций для двух рассмотренных моделей процесса эмиссии изопрена из океанических вод в атмосферу при наложении некоторого фиксированного функционального ограничения на искомую оптимальную функцию. Такое различие в выявленных оптимальных режимах эмиссии указывает на необходимость дальнейшего усовершенствования математических моделей потоков летучих органических соединений, исходящих из океанических вод.

\section{СПИСОК ЛИТЕРАТУРЫ}

1. Cropp, R. A. Coupling between ocean biota and atmospheric aerosols. Dust, dimethylsulphide or artifact? / R. A. Cropp, A. J. Garbic, G. H. McTainsh, R. D. Braddock // Global Biogeochemical cycles. 2005. - Vol. 19. -GB4002 (doi:10.1029/2004GB002436).

2. Gantt, B. A new physically - based quantification of marine isoprene and primary organic aerosol emissions/ B. Gantt, N. Meskhidze, D. Kamykowski // Atmos. Chem. Phys. - 2009. Vol. 9. - pp. 4915-4927.

3. Hackenberg, S. C. Potential controls of isoprene in the surface ocean / S.C. Hackenberg, S. J. Andrews, R. Airs, S. R. Arnold, H.A. Bouman, R.J. Brewin, R. J. Chance1, D. Cummings, G. Dall'Olmo, A. C. Lewis, J. K. Minaeian, K. M. Reifel, A. Small, G. A. Tarran, G. H. Tilstone, L. J. Carpenter // Global biogeochemical cycles. - 2017. - Vol. 31. - pp. 644-662 (Research article 10.1002/2016GB005531).

4. Kameyama, S. High-resolution observations of dissolved isoprene in surface seawater in the Southern Ocean during austral summer 2010-2011/ S. Kameyama, S. Yoshida, H. Tanimoto, S. Inomata, K. Suzuki, H. Yoshikawa-Inoue // J. Oceanogr. - 2014. - Vol. 70(3). pp. 225-239 (doi:10.1007/s10872-014-0226-8).

5. Kurihara, M. K. Distributions of short-lived iodocarbons and biogenic trace gases in the open ocean and atmosphere in the western North Pacific/ M.K. Kurihara // Mar. Chem. - 2010. - Vol. 118(3-4).pp. 156-170, (doi:10.1016/j.marchem.2009.12.001).

6. Lana, A. Potential for a biogenic influence on cloud microphysics over the ocean: a correlation study with satellite - derived data / A. Lana, R. Simo, S.M. Vallina, J. Dachs // Atmos. Chem. Phys. - 2012. vol. 12. - pp. 7977-7993 (doi:10.5194/acp-12-7977-2012). 


\section{ЭКОЛОГИЯ И ПРИРОДОПОЛЬЗОВАНИЕ}

7. Liakakoua, E. Isoprene above the Eastern Mediterranean: Seasonal variation and contribution to the oxidation capacity of the atmosphere / E. Liakakoua, M. Vrekoussisa, B. Bonsangb, Ch. Donousisa, M. Kanakidoua, N. Mihalopoulosa // Atmospheric Environment. - 2007. - Vol. 41. - pp. 1002-1010.

8. Ooki, A. A global-scale map of isoprene and volatile organic iodine in surface seawater of the Arctic, Northwest Pacific, Indian, and Southern Oceans/ A. Ooki, D. Nomura, S. Nishino, T. Kikuchi, Y. Yokouchi // J. Geophys. Res. Oceans. - 2015. Vol. 120-pp. 4108-4128 (doi:10.1002/2014JC010519).

9. Tran, S. A survey of carbon monoxide and non-methane hydrocarbons in the Arctic Ocean during summer 2010 / S. Tran, B. Bonsang, V. Gros, I. Peeken, R. Sarda-Esteve, A. Bernhardt, S. Belviso // Biogeosciences. - 2013. - Vol. 10(3). -pp. 1909-1935 (doi:10.5194/bg-10-1909-2013).

10. Wendy, J. Seasonal emissions of isoprene and other reactive hydrocarbon gases from the ocean / J. Wendy, P.S. Broadgate, A. Penkett // Geophysical research letters. - 1997. - vol. 24. - iss. 21.- pp. 2675 2678.

11. Zindler, C. Nutrient availability determines dimethyl sulfide and isoprene distribution in the eastern Atlantic Ocean / C. Zindler, C.A. Marandino, H.W. Bange, F. Schütte, E.S. Saltzman // Geophys. Res. Lett. - 2014. - vol. 41. - pp. 3181-3188 (doi:10.1002/ 2014GL059547).

\section{REFERENCES}

1. Cropp R.A., Garbic A.J., McTainsh G.H., Braddock R.D. Coupling between ocean biota and atmospheric aerosols. Dust, dimethylsulphide or artifact?, Global Biogeochemical cycles, 2005, vol. 19, GB4002 (doi:10.1029/2004GB002436).

2. Gantt B., Meskhidze N., Kamykowski D. A new physically - based quantification of marine isoprene and primary organic aerosol emissions, Atmos. Chem. Phys, 2009, vol. 9, pp. 4915-4927.

3. Hackenberg S.C., Andrews S.J., Airs R., Arnold S.R., Bouman H.A., Brewin R.J.W., Chance1 R.J., Cummings D., Dall' Olmo G., Lewis A.C., Minaeian J.K., Reifel K.M., Small A., Tarran G.A., Tilstone G.H., Carpenter L.J. Potential controls of isoprene in the surface ocean, Global biogeochemical cycles, 2017, Vol.31, pp. 644-662 (Research article 10.1002/2016GB005531).

4. Kameyama S., Yoshida S., Tanimoto H., Inomata S., Suzuki K., Yoshikawa-Inoue H. Highresolution observations of dissolved isoprene in surface seawater in the Southern Ocean during austral summer 2010-2011, J. Oceanogr, 2014, vol. 70(3), pp. 225-239 (doi:10.1007/s10872-014-0226-8).

5. Kurihara M. K., et al. Distributions of shortlived iodocarbons and biogenic trace gases in the open ocean and atmosphere in the western North Pacific, Mar. Chem, 2010, vol. 118(3-4), pp. 156-170, (doi:10.1016/j.marchem.2009.12.001).

6. Lana A., Simo R., Vallina S.M., Dachs J. Potential for a biogenic influence on cloud microphysics over the ocean: a correlation study with satellite - derived data, Atmos. Chem. Phys., 2012, vol. 12, pp. 7977-7993 (doi:10.5194/acp-12-7977-2012).

7. Liakakoua E., Vrekoussisa M., Bonsangb B., Donousisa Ch., Kanakidoua M., Mihalopoulosa N. Isoprene above the Eastern Mediterranean: Seasonal variation and contribution to the oxidation capacity of the atmosphere, Atmospheric Environment, 2007, vol. 41, pp. 1002-1010.

8. Ooki A., Nomura D., Nishino S., Kikuchi T., Yokouchi Y. A global-scale map of isoprene and volatile organic iodine in surface seawater of the Arctic, Northwest Pacific, Indian, and Southern Oceans, J. Geophys. Res. Oceans, 2015, vol. 120, pp. 4108-4128 (doi:10.1002/2014JC010519).

9. Tran S., Bonsang B., Gros V., Peeken I., SardaEsteve R., Bernhardt A., Belviso S. A survey of carbon monoxide and non-methane hydrocarbons in the Arctic Ocean during summer 2010, Biogeosciences, 2013, vol. 10(3), pp. 1909-1935 (doi:10.5194/bg-10-1909-2013).

10. Wendy J., Broadgate P.S., Penkett A. Seasonal emissions of isoprene and other reactive hydrocarbon gases from the ocean, Geophysical research letters, 1997, vol. 24, iss. 21, pp. 2675-2678.

11. Zindler C., Marandino C.A., Bange H.W., Schütte F., Saltzman E.S. Nutrient availability determines dimethyl sulfide and isoprene distribution in the eastern Atlantic Ocean, Geophys. Res. Lett., 2014, vol. 41, pp. 3181-3188 (doi:10.1002/ 2014GL059547). 


\section{Information about the Authors}

Hikmet Gamid ogly Asadov, Doctor of Technical Sciences, Professor, Head Department, Research Institute of Aerospace Informatics, National Aerospace Agency, S.S. Ahundova St., 1, AZ 1106 Baku, Azerbaijan, asadzade@rambler.ru.

Sima Azhdar gyzy Askerova, Ph. D. Candidate, Senior Researcher, Institute of Ecology, National Aerospace Agency, S.S. Ahundova St., 1, AZ 1106 Baku, Azerbaijan, asadzade@rambler.ru.

\section{Информация об авторах}

Хикмет Гамид оглы Асадов, доктор технических наук, профессор, начальник отделения НИИ Аэрокосмической информатики, Национальное аэрокосмическое агентство, ул. С.С. Ахундова, 1, AZ 1106 г. Баку, Азербайджан, asadzade@rambler.ru.

Сима Аждар гызы Аскерова, диссертант, старший научный сотрудник НИИ Экологии, Национальное аэрокосмическое агентство, ул. С.С. Ахундова, 1, AZ 1106 г. Баку, Азербайджан, asadzade@rambler.ru. 